\title{
Kin selection and the evolution of virulence
}

\author{
A Buckling ${ }^{1}$ and MA Brockhurst ${ }^{2}$ \\ ${ }^{1}$ Department of Zoology, University of Oxford, Oxford, UK and ${ }^{2}$ School of Biological Sciences, Biosciences Building, University of \\ Liverpool, Liverpool, UK
}

\begin{abstract}
Social interactions between conspecific parasites are partly dependent on the relatedness of interacting parasites (kin selection), which, in turn, is predicted to affect the extent of damage they cause their hosts (virulence). High relatedness is generally assumed to favour less competitive interactions, but the relationship between relatedness and virulence is crucially dependent on the social behaviour in question. Here, we discuss the rather limited body of experimental work that addresses how kin-selected social behaviours affect virulence. First, if prudent use of host resources (a form of cooperation) maximizes the transmission success of the parasite population, decreased relatedness is predicted to result in increased host exploitation and virulence. Experimental support for this well-established theoretical result is surprisingly limited. Second, if parasite within-host
\end{abstract}

growth rate is a positive function of cooperation (that is, when individuals need to donate public goods, such as extracellular enzymes), virulence is predicted to increase with increasing relatedness. The limited studies testing this hypothesis are broadly consistent with this prediction. Finally, there is some empirical evidence supporting theory that suggests that spiteful behaviours are maximized at intermediate degrees of relatedness, which, in turn, leads to minimal virulence because of the reduced growth rate of the infecting population. We highlight the need for further thorough experimentation on the role of kin selection in the evolution of virulence and identify additional biological complexities to these simple frameworks.

Heredity (2008) 100, 484-488; doi:10.1038/sj.hdy.6801093; published online 23 January 2008

Keywords: experimental evolution; parasite; cooperation; spite; microbe; public goods

\section{Introduction}

Sociality appears to be ubiquitous throughout the domains of life (Maynard-Smith and Szathmary, 1995; West et al., 2007). Recent years have seen growing interest in understanding social evolution in microbes (Crespi, 2001; Travisano and Velicer, 2004; West et al., 2006; Foster et al., 2007), probably for two major reasons: first, microbes offer very tractable experimental systems for studying the ecology and evolution of social traits in real time and under carefully controlled conditions. Second, microbial sociality has important applied implications. The focus of this article is the impact of social interactions between conspecific microbial pathogens on disease virulence (that is, the damage caused to hosts).

The most obvious social trait is cooperation (that is, actions that benefit individuals other than the actor). We distinguish between two forms of cooperation: (1) Prudence, where individuals limit their resource use for the benefit of others and (2) Public goods production, where individuals donate resources that are of benefit to others. Why should cooperation exist, given that noncooperating cheats are likely to be able to exploit their cooperative neighbours and therefore have a short-term selective advantage? There are two general explanations

Correspondence: $\operatorname{Dr}$ A Buckling, Department of Zoology, Oxford University, Department of Plant Science, University of Oxford, South Parks Road, Oxford OX1 3RB, Oxford OX1 3PS, UK.

E-mail: angus.buckling@zoo.ox.ac.uk

Received 29 May 2007; revised 9 October 2007; accepted 28 October 2007; published online 23 January 2008 for the evolution of cooperation, and they apply to all taxa (Hamilton, 1964; Sachs et al., 2004; Lehmann, 2006; West et al., 2007). First, cooperation might provide a direct fitness benefit to the actor. This would be the case if, for example, individuals can choose to cooperate only with those individuals who reciprocate the cooperation; or if the interests of two parties are directly aligned; or if cheating behaviour is punished. Second, cooperation might provide an indirect fitness benefit to the actor by promoting the transmission of copies of its genes that are carried by its relatives. The genes for cooperation are favoured if they are successfully transmitted, and it does not matter whether the actor or the recipient transmits them (inclusive fitness; Hamilton, 1964). Kin selection is the process that maximizes inclusive fitness (Maynard-Smith, 1964). For kin selection to favour cooperation, the degree of genetic relatedness of interacting individuals must be high relative to the population as a whole. Relatedness is a statistical measure of genetic similarity and refers specifically to the cooperative loci in question (Hamilton, 1964); however, high relatedness at one locus is likely to show on average a positive correlation with relatedness at other loci across the whole genome (Grafen, 2006).

A less obvious intraspecific social trait (although potentially as important as cooperation with respect to virulence) is spite (Hamilton, 1970; Gardner and West, 2004). Spite is costly to both the actor and the recipient. Given the direct fitness costs, spiteful behaviours can only be explained by kin selection. Spiteful behaviours can be favoured when the recipient of the behaviour has a lower relatedness to the actor than the population as a 
whole, and when these 'negatively related' individuals can be specifically targeted by the spiteful action. The actor then indirectly benefits from the spiteful behaviour by reducing competition experienced by the actor's relatives.

In this article, we specifically focus on experimental data that address how kin-selected cooperation and spite affect virulence. The reason for this focus is that kin selection is crucially dependent on parasite ecology and epidemiology. In the broadest terms, the extent of parasite cooperation and spite will depend on whether or not parasitic infections comprised single or multiple genotypes.

\section{Relatedness and virulence}

How relatedness, through its effect on cooperative and spiteful traits, is predicted to affect virulence depends on the type of intraspecific social behaviour displayed by parasites. Here, we distinguish between three types of social behaviours: (1) prudence; (2) public goods cooperation and (3) spite.

\section{Prudence}

Virulence is often assumed to be a necessary consequence of host exploitation and hence transmission to new hosts (parasite fitness) (Anderson and May, 1982; Bremermann and Pickering, 1983; Nowak and May, 1994; Frank, 1996; Ebert and Bull, 2003). Virulence also has a negative impact on transmission success, for example, if parasites can only transmit from live hosts or if transmission is reduced from debilitated hosts. As a result of this trade-off, some intermediate level of host exploitation (optimal virulence) will maximize the transmission success of the parasite population. The relatedness of coinfecting parasite genotypes is also likely to affect the virulence of infections; kin-selection models predict that the level of parasite virulence that maximizes parasite fitness increases with decreasing relatedness of coinfecting parasites (Bremermann and Pickering, 1983; Nowak and May, 1994; Frank, 1996) (Figure 1a). This arises because optimal virulence is displayed only if individuals exploit host resources prudently, and it is predicted to occur when relatedness between coinfecting parasite genotypes is high. When relatedness is low, competition between parasites with unrelated genotypes within a host results in selection of individuals that use resources as quickly as possible, thus increasing virulence despite reducing group success, an idea epitomized by Hardin's 'tragedy of the commons' (Hardin, 1968; Frank, 1998).

Some studies in natural populations are consistent with the prudence model. When measured across closely related species, the virulence of parasitic nematodes that infect fig wasps increases with increasing average number of foundresses that lay their eggs in a single fig (Herre, 1993). This result has been interpreted in terms of kin selection: more foundresses increase the chance of mixed genotype nematode infections, and hence the evolution of higher virulence (Herre, 1993; Frank, 1996). The correlational nature of the data means that they can be interpreted in other ways, and hence the need for experimental tests.

Experimental evidence for the negative relationship between relatedness and virulence is limited (Brown et al., 2002; West and Buckling, 2003). Indeed, in studies where decreased relatedness results in increased virulence, there is little evidence to suggest that it is the result of increased within-host exploitation at the expense of between-host transmission. For example, mixed genotype infections of rodent malaria parasites (Plasmodium chabaudi) infecting mice were more virulent (in terms of host weight loss and anaemia) than single clone infections, but did not show elevated rates of reproduction or density (Taylor et al., 1998). This suggests that the immune system was less able to control the infections because of their antigenic diversity, rather than increased virulence, resulting from increased host exploitation. Similarly, mixed infections of caterpillars (Panolis flammea) with baculoviruses reached higher virulence, but in this system host death is required for transmission; hence, the classic transmission-virulence trade-off does not apply and there is no cost to virulence (Hodgson et al., 2004). However, in this and other obligate-killing parasites, there is likely to be a trade-off between time-to-kill and progeny yield, as prudence (that is, low rates of host exploitation) can maximize progeny yield and, by extension, transmission opportunities (Hodgson et al., 2004). No evidence for a difference in time to death between single and mixed genotype infections was detected. A further study of schistosomes (Schistosoma mansoni) infecting snails (Biomphalaria glabrata) reported elevated total reproduction rate in mixed versus single genotype infections, but it was impossible to distinguish whether this was due to individual strains replicating faster or again due to the immune system's not being good at controlling mixed genotype infections (Davies et al., 2002).
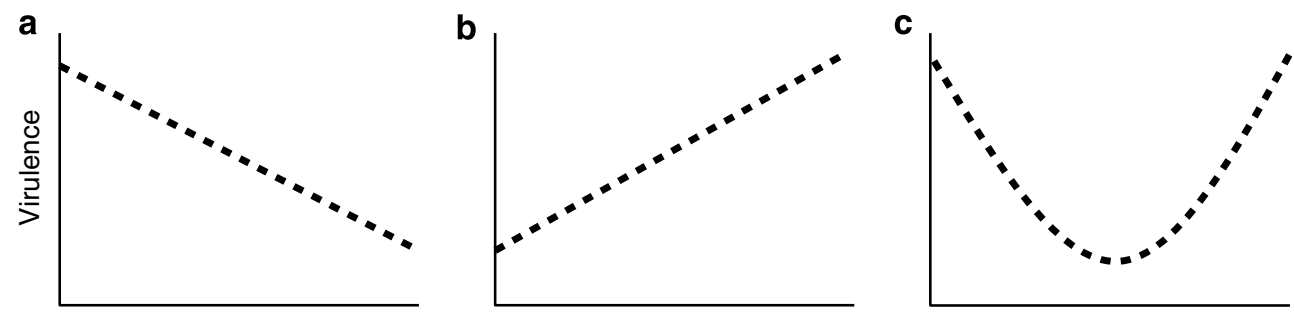

Relatedness

Figure 1 Hypothesized relationships between virulence and relatedness under conditions of prudence (a), public goods cooperation (b) and spiteful interactions (c). 
There are many probable reasons why the data are generally inconsistent with the prudence model, the most obvious being that the model assumptions are often inappropriate for the particular host-parasite system. These issues have been addressed in detail elsewhere (Read and Taylor, 2001; Ebert and Bull, 2003; Lambrechts et al., 2006), and we limit further discussion of the importance of biological details to different types of social interactions (see below). However, another contributing factor is that the vast majority of experiments testing the model have been short-term ecological studies, where infections have been established with either single or several parasite genotypes. Providing experimental support for prudence therefore requires that parasites are able to facultatively upregulate their reproductive rates upon detection of another parasite genotype in the same host. We are unaware of any evidence demonstrating that this occurs.

An alternative approach to test the theory would be to experimentally evolve parasites under conditions of high and low relatedness, and then determine the virulence of the evolved lines. The only experiment we are aware of, which may have done this, was designed for a different purpose: to address the effect of extrinsic host mortality of Daphnia magna on the evolution of virulence of a horizontally transmitted microsporidian parasite (Glugoides intestinalis) (Ebert and Mangin, 1997). It was predicted that higher virulence should be favoured in high mortality treatment, because of the reduced cost to the parasite of killing the host early. The opposite result was observed. A possible explanation is that the high host mortality treatment reduced the chances of mixed infections, and hence within-host competition, as extrinsically 'killed' (removed) hosts were replaced with uninfected hosts (Ebert and Mangin, 1997; Gandon et al., 2001). This interpretation is supported by the large number of experiments in which parasite virulence increases following serial transfer from one host to the other (reviewed in Ebert, 1998). Under these conditions, natural selection will favour parasites that have the highest within-host competitive ability. These data are therefore strongly suggestive of a positive genetic correlation between within-host competitive ability and virulence, a crucial assumption of the prudence model. Studies that have attempted to directly measure genetic correlations are less consistent, with both positive (de Roode et al., 2005) and negative (Gower and Webster, 2005) correlations reported for P. chabaudi and S. mansoni, respectively. Many more experiments are required to fully test the evolutionary impact of parasite relatedness on within-host prudence.

\section{Public goods cooperation}

In contrast to models that assume that prudence maximizes transmission, a positive relationship between relatedness and virulence is predicted if host exploitation is dependent on parasites cooperating with each other (Chao et al., 2000; Brown et al., 2002; West and Buckling, 2003) (Figure 1b). Such situations arise if parasites produce some form of public good that can be exploited by nonproducing cheats. The limited experimental work directly addressing the role of relatedness in determining parasite virulence under public goods cooperation (as well as observational examples; see Chao et al., 2000) is broadly consistent with the theory.

Turner and Chao (1999) evolved lytic phages on bacteria (Pseudomonas syringae) under conditions of high and low relatedness (low and high multiplicity of infection, respectively) and found that a correlate of virulence (viral competitive fitness) evolved to be much lower under low relatedness. Bacteriophages produce replicative enzymes that can be used by all virions in the cell and, as such, can be considered a public good. Under low relatedness, when multiple infection was likely, cheating mutants that did not pay the metabolic cost of producing replication enzymes evolved and had a selective advantage when coinfecting with replicase-producing cooperators. By contrast, under conditions of high relatedness, cheats did not evolve because they could not share cells with cooperators.

Another reasonably well-studied example of public good cooperation is the production of extracellular iron-scavenging molecules (siderophores) by the opportunistic bacterial pathogen, Pseudomonas aeruginosa. Siderophore production is beneficial when iron is limiting, but it is also metabolically costly. Consequently, in mixed populations where both wild-type and mutant bacteria are present, the mutants can gain the benefit of siderophore production without paying the cost and hence increase in frequency, as they outcompete the cooperative wild type (Griffin et al., 2004). Consistent with the above in vitro results, siderophore-negative cheats have been observed in natural populations of $P$. aeruginosa that have infected the lungs of cystic fibrosis patients (de Vos et al., 2001).

The role of relatedness in siderophore-mediated cooperation and virulence was addressed by inoculating caterpillars (Galleria mellonella) with single clones of a wild-type, siderophore-producing strain of $P$. aeruginosa (cooperators), an isogenic siderophore-minus mutant (cheats), or both (Harrison et al., 2006). Cheats grew more rapidly in mixed, as opposed to single-clone infections, whereas the opposite pattern was observed for cooperators. Low-relatedness infections are therefore more likely to favour the evolution of siderophore cheats than are high-relatedness infections (Harrison et al., 2006). Infections containing cheats were less virulent than pure cooperator infections, as has been observed in a previous study (Meyer et al., 1996). Hence, low-relatedness infections are less virulent.

However, in these simple competition experiments between siderophore cheats and cooperators, cheats never had a detectable selective advantage: at best, their fitness was the same as that of cooperators (Harrison et al., 2006). This is in contrast with in vitro work where cheats were consistently fitter under iron-limited conditions (Griffin et al., 2004). This poses a major problem for the theory: if cheats never have a selective advantage in vivo, then virulence will not decrease under lowrelatedness infections. We have, however, since observed that cheats do indeed evolve de novo and increase in frequency when passaged through caterpillars (Racey, Harrison and Buckling, in prep).

Recent in vitro work suggests that the positive relationship between relatedness and virulence where public goods determines growth rate may be more complex (Brockhurst et al., 2006). Pathogenic bacteria often exist as biofilms - a group of bacteria attached to surfaces and 
covered in polymer. Biofilm living confers a group advantage, for example, in terms of resistance to antibiotics and immune attack. The biofilm polymer is another example of a public good, and nonproducing cheats are likely to have a selective advantage under conditions of mixed genotypes and low relatedness. As such, mixed genotype infections should result in the breakdown of cooperation and reduced virulence. However, the diversity with respect to cooperative traits is also likely to correlate with diversity in other traits, such as resource use. A recent study found that biofilms made up of mixtures of genotypes that were specialized on different resources, as a result of fitness trade-offs, were more resilient to invasion by cheats than biofilms consisting of a single genotype, presumably because cheats also traded off resource use, and hence were better at cheating on one cooperating genotype than another. As such, selection against cooperation, resulting from the reduction in relatedness (by definition, at the cooperative locus) in mixed infections, can potentially be opposed by increased niche partitioning, which can limit the invasion of cheats, and also increase population density because of more efficient resource use (Brockhurst et al., 2006). This could weaken or even eliminate the relationship between virulence and relatedness.

\section{Spite}

If spiteful behaviours are a key determinant in the outcome of competition between parasite lineages, then virulence is predicted to peak at high and low levels of relatedness (Figure 1c) (Gardner et al., 2004). This is because spiteful behaviours reduce the total population growth rate and are also predicted to be maximized at intermediate levels of relatedness. Under high levels of relatedness, there is little benefit in harming close relatives, and hence spiteful behaviours are not favoured. Under very low relatedness, a lineage will necessarily be at a low frequency and thus will not be able to affect all competitors; hence, the benefit of the spiteful behaviour in terms of harming competitors and freeing up resources will be experienced by non-kin as much as kin. It is only when a spiteful lineage is at a high enough frequency, and there are sufficient competitors to harm (intermediate relatedness) that the inclusive fitness benefits of spite will be realized.

Anticompetitor toxins produced by bacteria (bacteriocins) are a good example of spiteful behaviours. Bacteriocins are costly to produce (often involving cell lysis for their release) and are capable of killing susceptible competitors. Bacteriocin genes are generally linked to appropriate immunity genes, rendering close relatives immune (Riley and Wertz, 2002). We are aware of only one study that has addressed the role of 'relatedness' in virulence where spiteful behaviours determine competitive outcomes (Massey et al., 2004). Different strains of the insect pathogenic bacteria Photorhabdus and Xenorhabdus spp. were inoculated into caterpillars as single- or mixed-clone infections. Virulence was reduced in mixed infections relative to single infections where both strains could kill each other. Furthermore, there was a large competitive advantage to being able to produce bacteriocins only when in mixed infections. This suggests that infections consisting of two genotypes favour bacteriocin production relative to single genotype infections and that bacteriocin production reduces virulence. Note that different strains in this study are probably more distantly related to each other than is typically assumed for a single 'species'.

\section{Virulence and the scale of competition}

Kin-selected cooperation can be modulated by the scale of competition (Taylor, 1992; West et al., 2002). Most organisms, including parasites, exist in metapopulations, that is, the population is made up of loosely connected patches of individuals (a patch being a host or a group of hosts), and most interactions occur within these patches. If successful patches (that is, those containing a high frequency of cooperating individuals) contribute more progeny to the whole metapopulation (there is competition between patches, global competition), then cooperation is more likely to be favoured across the metapopulation as a whole. However, if competition is local, occurring only within patches (that is, the output of the patch is independent of patch success), then cooperative strategies will not be favoured. Even if the relatedness within patches is very high, cooperation will not be favoured by selection when competition is local because helping some kin effectively hinders others (West et al., 2002). The impact of the scale of competition (and relatedness, in a fully factorial design) on cooperation was experimentally addressed using $P$. aeruginosa siderophore production in vitro (Griffin et al., 2004) As predicted, high relatedness and global competition favoured siderophore producers, whereas low relatedness and local competition favoured nonproducing cheats. How the scale of competition affects virulence depends on the type of social behaviour. Local competition favours public goods cheats and hence low virulence (West and Buckling, 2003). Similarly, it will favour spiteful behaviours, which only affect local competitors, and hence low virulence (Gardner et al., 2004). In contrast, local competition will select for less prudence and hence increased virulence. No experiments to date have explicitly manipulated the scale of competition to determine its effect on virulence, but results of experiments involving serial host-to-host transfer of parasites (Ebert, 1998) are consistent with the theory. In these studies, competition is entirely local (selection acts only within hosts) and cooperation, in the form of prudence, appears to break down, resulting in increased virulence.

\section{Conclusions}

There is a small but growing body of evidence that kin-selection-driven social traits can play an important role in the evolution of virulence. Much of these data result from studying the ecology and real-time evolution of microbes under controlled laboratory conditions, but note that extrapolation of in vitro studies and in vivo studies using unnatural host to the real world must be done with caution. How relatedness affects virulence crucially depends on the type of social interaction. In simple terms, a negative relationship is expected if cooperation is prudent resource use; a positive relationship if cooperation is the production of public goods; and an inverse unimodal if competition is mediated by spiteful behaviours. However, these relationships can be altered by other factors. For example, low relatedness 
may correlate with increase niche partitioning, which can increase the growth rate and hence virulence of populations. Furthermore, all three broad categories of social interaction may operate simultaneously, leading to even more complex patterns. Finally, it is necessary to emphasize that virulence evolution is likely to be affected by many additional selective forces imposed by the ecology of the host and parasite. Furthermore, the expression of virulence is by no means solely determined by parasites, but rather by interactions between host and parasite genotypes (Read and Taylor, 2001; Ebert and Bull, 2003; Lambrechts et al., 2006).

\section{Acknowledgements}

We thank Michael Bonsall, Stu West and Ben Raymond for comments on the article. AB is supported by the Royal Society and Leverhulme Trust.

\section{References}

Anderson RM, May RM (1982). Coevolution of hosts and parasites. Parasitology 85: 411-426.

Bremermann HJ, Pickering J (1983). A game-theoretical model of parasite virulence. J Theor Biol 100: 411-426.

Brockhurst MA, Hochberg ME, Bell T, Buckling A (2006). Character displacement promotes cooperation in bacterial biofilms. Curr Biol 16: 2030-2034.

Brown SP, Hochberg ME, Grenfell BT (2002). Does multiple infection select for raised virulence? Trends Microbiol 10: 401-405.

Chao L, Hanley KA, Burch CL, Dahlberg C, Turner PE (2000). Kin selection and parasite evolution: Higher and lower virulence with hard and soft selection. $Q$ Rev Biol 75: 261-275.

Crespi BJ (2001). The evolution of social behavior in microorganisms. Trends Ecol Evol 16: 178-183.

Davies CM, Fairbrother E, Webster JP (2002). Mixed strain schistosome infections of snails and the evolution of parasite virulence. Parasitology 124: 31-38.

de Roode JC, Pansini R, Cheesman SJ, Helinski MEH, Huijben S, Wargo AR et al. (2005). Virulence and competitive ability in genetically diverse malaria infections. Proc Natl Acad Sci USA 102: 7624-7628.

de Vos D, De Chial M, Cochez C, Jansen S, Tummler B, Meyer JM et al. (2001). Study of pyoverdine type and production by Pseudomonas aeruginosa isolated from cystic fibrosis patients: prevalence of type II pyoverdine isolates and accumulation of pyoverdine-negative mutations. Arch Microbiol 175: 384-388.

Ebert D (1998). Experimental evolution of parasites. Science 282 1432-1435.

Ebert D, Bull JJ (2003). Challenging the trade-off model for the evolution of virulence: is virulence management feasible? Trends Microbiol 11: 15-20.

Ebert D, Mangin KL (1997). The influence of host demography on the evolution of virulence of a microsporidian gut parasite. Evolution 51: 1828-1837.

Foster KR, Parkinson K, Thompson CRL (2007). What can microbial genetics teach sociobiology? Trends Genetics 23: 74-80.

Frank SA (1996). Models of parasite virulence. $Q$ Rev Biol 71: 37-78.

Frank SA (1998). Foundations of Social Evolution. Princeton University Press: Princeton, NJ.

Gandon S, Jansen VAA, van Baalen M (2001). Host life history and the evolution of parasite virulence. Evolution 55: 1056-1062.

Gardner A, West SA (2004). Spite and the scale of competition. J Evol Biol 17: 1195-1203.
Gardner A, West SA, Buckling A (2004). Bacteriocins, spite and virulence. Proc $R$ Soc Lond B 271: 1529-1535.

Gower CM, Webster JP (2005). Intraspecific competition and the evolution of virulence in a parasitic trematode. Evolution 59: 544-553.

Grafen A (2006). Optimisation of inclusive fitness. J Theor Biol 238: 541-563.

Griffin AS, West SA, Buckling A (2004). Cooperation, competition in pathogenic bacteria. Nature 430: 1024-1027.

Hamilton WD (1964). The genetical evolution of social behaviour, I and II. J Theor Biol 7: 1-52.

Hamilton WD (1970). Selfish and spiteful behaviour in an evolutionary model. J Evol Biol 228: 1218-1220.

Hardin G (1968). The tragedy of the commons. Science 162: 1243-1248.

Harrison F, Browning LE, Vos M, Buckling A (2006). Cooperation and virulence in acute Pseudomonas aeruginosa infections. BMC Biology 4: 21.

Herre EA (1993). Population-structure and the evolution of virulence in nematode parasites of fig wasps. Science 259: $1442-1445$.

Hodgson DJ, Hitchman RB, Vanbergen AJ, Hails RS, Possee RD, Cory JS (2004). Host ecology determines the relative fitness of virus genotypes in mixed-genotype nucleopolyhedrovirus infections. J Evol Biol 17: 1018-1025.

Lambrechts L, Fellous S, Koella JC (2006). Coevolutionary interactions between host and parasite genotypes. Trends Parasitol 22: 12-16.

Lehmann L, Keller L (2006). The evolution of cooperation and altruism. A general framework and classification of models. J Evol Biol 19: 1365-1378.

Massey RC, Buckling A, Ffrench-Constant R (2004). Interference competition and parasite virulence. Proc $R$ Soc Lond B 271: 785-788.

Maynard-Smith J, Szathmary E (1995). The Major Transitions in Evolution. WH Freeman: Oxford.

Maynard-Smith J (1964). Group selection and kin selection. Nature 201: 1145-1147.

Meyer JM, Neely A, Stintzi A, Georges C, Holder IA (1996). Pyoverdin is essential for virulence of Pseudomonas aeruginosa. Infect Immun 64: 518-523.

Nowak MA, May RM (1994). Superinfection and the avolution of parasite virulence. Proc Natl Acad Sci USA 255: 81-89.

Read AF, Taylor LH (2001). The ecology of genetically diverse infections. Science 292: 1099-1102.

Riley MA, Wertz JE (2002). Bacteriocins: evolution, ecology, and application. Ann Rev Microbiol 56: 117-137.

Sachs JL, Mueller UG, Wilcox TP, Bull JJ (2004). The evolution of cooperation. Q Rev Biol 79: 135-160.

Taylor LH, Mackinnon MJ, Read AF (1998). Virulence of mixed-clone and single-clone infections of the rodent malaria Plasmodium chabaudi. Evolution 52: 583-591.

Taylor PD (1992). Altruism in viscous populations-an inclusive fitness model. Evol Ecol 6: 352-356.

Travisano M, Velicer GJ (2004). Strategies of microbial cheater control. Trends Microbiol 12: 72-78.

Turner PE, Chao L (1999). Prisoner's dilemma in an RNA virus. Nature 398: 441-443.

West SA, Buckling A (2003). Cooperation, virulence and siderophore production in bacterial parasites. Proc $R$ Soc Lond B 270: 37-44.

West SA, Griffin AS, Gardner A (2007). Social semantics: altruism, cooperation, mutualism, strong reciprocity and group selection. J Evol Biol 20: 415-432.

West SA, Griffin AS, Gardner A, Diggle S (2006). Social evolution theory for microbes. Nat Rev Microbiol 4: 597-607.

West SA, Pen I, Griffin AS (2002). Conflict and cooperationcooperation and competition between relatives. Science 296: 72-75. 